[3] Song J.S., Do J.H., and Lee S.W.: The prevalence and the clinical relevance of anti-Ro52 in Korean patients with primary Sjogren's syndrome. Rheumatol Int 2011

[4] Salomonsson S, Jonsson MV, Skarstein K, et al. Cellular basis of ectopic germinal center formation and autoantibody production in the target organ of patients with Sjogren's syndrome. Arthritis Rheum. 2003

[5] Imgenberg-Kreuz J, AC, Nordmark G, et al. Transcription profiling of peripheral $B$ cells in antibody-positive primary Sjögren's syndrome reveals upregulated expression of CX3CR1 and a type I and type II interferon signature. Scand J Immunol. 2018

Acknowledgement: Center for Genes,Environment and Health Ms. Kendra Walton

Disclosure of Interests: None declared

DOI: 10.1136/annrheumdis-2019-eular.707

\section{AB0179 THE TRANSCRIPTION FACTORS IKZF1 AND IKZF3 CONTROL B CELL ACTIVATION AND DIFFERENTIATION IN SYSTEMIC LUPUS ERYTHEMATOSUS}

Sotiria Manou-Stathopoulou ${ }^{1}$, Felice Rivellese ${ }^{1}$, Daniele Mauro ${ }^{1}$, Katriona Goldmann ${ }^{1}$, Debasish Pyne ${ }^{2}$, Peter Schafer ${ }^{3}$, Michele Bombardieri ${ }^{1}$, Costantino Pitzalis ${ }^{1}$, Myles Lewis ${ }^{1} .{ }^{1}$ William Harvey Research Institute, Queen Mary University of London, Experimental Medicine and Rheumatology, London, United Kingdom, ${ }^{2}$ Barts Health NHS Trust, Rheumatology, London, United Kingdom; ${ }^{3}$ Celgene Corporation, Summit, United States of America

Background: The transcription factors IKZF1 (Ikaros) and IKZF3 (Aiolos), essential for the maturation, differentiation and survival of $B$ cells, have been linked to Systemic Lupus Erythematosus (SLE). The cereblon modulator Iberdomide, which induces degradation of IKZF1 and IKZF3, is undergoing clinical trials in SLE. However, the role of IKZF1 and IKZF3 in aberrant plasmablast development and pathogenesis of SLE has not been fully elucidated.

Objectives: To assess the mechanism of IKZF1 and IKZF3 control of gene expression underlying activation and differentiation of $B$ cells in SLE patients.

Methods: CD19 ${ }^{+} \mathrm{B}$ cells were isolated from the peripheral blood of patients with SLE recruited at Barts Health NHS Trust $(n=25)$. B cells were cultured for 5 days and stimulated with IL-2, IL-10, IL-15, CD40L and TLR-7 ligand Resiquimod to induce plasmablast differentiation together with iberdomide $(10 \mathrm{nM})$ or control at either day 0 or $18 \mathrm{~h}$ prior to harvest at day 4 . At day 5 , cells were harvested for fluorescence-activated cell sorting (FACS) and measurement of $\lg G$ and $\lg M$ in supernatants by ELISA. RNA extraction and RNA-sequencing were performed on FACS-sorted CD27- $\operatorname{lgD}{ }^{+}$naïve $\mathrm{B}$ cells and $\mathrm{CD} 20^{\text {low }} \mathrm{CD} 27^{+} \mathrm{CD} 38^{+}$plasmablasts and matched baseline $B$ cells.

Results: Iberdomide from day $0(n=9)$ significantly reduced the number of $\mathrm{CD} 20^{\text {low }} \mathrm{CD} 27^{+} \mathrm{CD} 38^{+}$plasmablasts (number of sorted $\mathrm{CD} 20^{\text {low }} \mathrm{CD} 27^{+} \mathrm{CD} 38^{+}$ plasmablasts mean \pm SD $48727 \pm 86122$ in untreated, $3248 \pm 8335$ in iberdomide, $p=0.03$ ). Accordingly, iberdomide significantly inhibited the production of $\lg M$ and IgG from SLE B cells ( $p=0.017$ and 0.050 , respectively). Iberdomide given at day 4 did not affect the numbers of plasmablasts or the production of $\lg M$ and $\operatorname{lgG}$ $(n=16)$. However, iberdomide induced a significant modulation of several genes both in naïve $B$ cells and plasmablasts, as assessed by RNA-seq on sorted cells (400 and 461 differentially modulated genes in naïve $B$ cells and plasmablasts, $p$ adjusted $<0.05$ ). Pathway analysis showed that iberdomide treatment resulted in downregulation of JAK-STAT signalling downstream of IL12 (FDR=7.92E-04), IL12 signalling (FDR0.0014), and p53 signalling regulation of cell death (FDR=0.0043) and showed a trend to upregulation of RUNX1 signalling and Rho GTPase cycle.

Conclusion: Our work confirms the importance of IKZF1 and IKZF3 in modulating gene expression required for B cell differentiation in SLE, as shown by the ability of iberdomide to inhibit the differentiation of SLE B cells into plasmablasts. Inhibition of IKZF1 and IKZF3 modulated the expression of several transcriptional programmes in naïve $B$ cells and plasmablasts. While larger scale analysis will be needed to confirm the functional consequences of IKZF1 and IKZF3 modulation in naïve $B$ cells and plasmablasts, our results confirm their relevance in SLE as important regulators of $B$ cell activation and differentiation.

Acknowledgement: This study was supported by research funding from Celgene Corporation.

Disclosure of Interests: Sotiria Manou-Stathopoulou: None declared, Felice Rivellese: None declared, Daniele Mauro: None declared, Katriona Goldmann: None declared, Debasish Pyne: None declared, Peter Schafer Shareholder of: Celgene corporation, Employee of: Celgene Corporation, Michele Bombardieri Grant/research support from: Celgene, Consultant for: Medimmune, Costantino Pitzalis Grant/research support from: Celgene, Myles Lewis Grant/research support from: Celgene

DOI: 10.1136/annrheumdis-2019-eular.6343

\section{AB0180 \\ VALIDATION OF COMPLEX IMMUNOPHENOTYPING STRATIFICATION OF PATIENTSWITH LUPUS AND SJÖGREN'S SYNDROME WITH THERAPEUTIC POTENTIAL}

Lucia Martin-Gutierrez ${ }^{1,2}$, Hannah Peckham ${ }^{1}$, George Robinson ${ }^{1,2}$, Giulia Varnier ${ }^{1}$, Elizabeth Jury ${ }^{1}$, Coziana Ciurtin ${ }^{2} .{ }^{1}$ UNIVERSITY COLLEGE OF LONDON, Rheumatology, LONDON, United Kingdom; ${ }^{2}$ UNIVERSITY COLLEGE OF LONDON, Arthritis Research UK Centre for Adolescent Rheumatology at UCLH, UCL and Great Ormond Street Institute of Child Health, LONDON, United Kingdom

Background: There is a current paucity in effective treatments for patients with primary Sjögren's syndrome (pSS) and systemic lupus erythematosus (SLE). Their overlapping symptoms mean that they are often treated with similar biologic therapeutics, despite being distinct diagnoses. Our pilot research sought to stratify patients by novel, complex immune signatures, to potentially facilitate a more personalised approach to prescribing medication, irrespective of clinical diagnosis. From a cohort of 40 patient patients with SLE, pSS or combined SS/SLE, and 33 healthy controls; five distinct clusters were originally identified.

Objectives: Here we aimed to validate the original patient endotypes from our pilot data with a new patient cohort, and refine the identified clusters to be of more realistic clinical benefit.

Methods: A new cohort of patients with SLE $(n=32)$, pSS $(n=31)$ and SS/SLE $(n=15)$ donated peripheral blood samples while attending appointments at UCLH clinics. None had received B-cell depletion therapy in the preceding 48 months. Complex phenotyping of peripheral $\mathrm{T}$ and $\mathrm{B}$-cell subsets was performed by flow cytometry. Unsupervised clustering, statistical comparison to healthies and Receiver Operator Characteristic (ROC) analysis, as per our previous work, were used to stratify patients using immune signatures into distinct endotypes consider ing only those markers where sensitivity and specificity were above $80 \%$ (AUC> 0.80 ). Characteristics of new clusters were compared with previous findings to refine the number of markers and unique groups.

Results: Five distinct endotypes across the three disease groups were identified after unsupervised clustering of CD19+ B-cell, CD4+ and CD8+ T-cell subsets Two of these clusters serve to validate groupings from our original data- one with a distinct $B$ cell fingerprint $(\mathrm{Bm} 1, \mathrm{IBm5}$ and Total $\mathrm{CD} 19+)$ with $\mathrm{AUC}=0.87$, $\mathrm{AUC}=0.81$ and $\mathrm{AUC}=0.83$ respectively, and another with a $\mathrm{T}$ cell fingerprint $($ Total CD4+, Total CD8+, CD8+ naïve, CD8+ effector memory T cell subsets) with $A \cup C>0.80$ for all of them. Clinical diagnoses and symptoms treatment did not differ significantly between clusters.

Conclusion: Our results demonstrate a novel method to re-classify patients using clustered immune phenotype abnormalities. Two distinct groups demonstrated either B or T-cell immunophenotypes, likely reflective of their underlying immunopathogenesis. Stratification by patient endotype has the potential to facilitate a better-informed selection of biologic therapeutics for individual patients; and highlights pathways for further mechanistic research.

Acknowledgement: British Sjögren's syndrome Association and Versus Arthritis Disclosure of Interests: None declared

DOI: 10.1136/annrheumdis-2019-eular.5696

\section{AB0181 ASSOCIATION BETWEEN SINGLE NUCLEOTIDE POLYMORPHISMS (SNPS) OF THE BAFF GENE AND FATIGUE IN PRIMARY SJÖGREN'S SYNDROME}

Christina Maria Flessa ${ }^{1}$, Adrianos Nezos ${ }^{1}$, Evangelia Zampeli ${ }^{2}$, Haralampos M. Moutsopoulos ${ }^{3}$, Clio Mavragani ${ }^{1,2,4}$. ${ }^{1}$ Medical School, National and Kapodistrian University of Athens, Department of Physiology, Athens, Greece; ${ }^{2}$ Medical School, National and Kapodistrian University of Athens, Department of Pathophysiology, Athens, Greece; ${ }^{3}$ Academy of Athens, Athens, Greece; ${ }^{4}$ National and Kapodistrian University of Athens, School of Medicine, Joint Academic Rheumatology Program, Athens, Greece

Background: Primary Sjögren's Syndrome (SS) is characterized by B lymphocyte hyperactivity with B cell activating factor (BAFF) acting as an important regulator. Single Nucleotide Polymorphisms (SNPs) of the BAFF gene have been implicated in the pathogenesis of several autoimmune diseases including lupus and SS both of which are characterized by heightened fatigue levels, often compromising quality of life.

Objectives: To explore potential associations between several BAFF gene SNPs and fatigue status experienced by primary SS patients.

Methods: Fatigue status was assessed by Functional Assessment of Chronic IIIness Therapy-Fatigue (FACIT-F) scale in 178 primary SS patients. Five SNPs (rs9514827, rs1041569, rs9514828, rs1224141, rs12583006) of the BAFF gene were tested in DNA extracted from peripheral blood of all patients enrolled in the study using the RFLP-PCR method. A cut-off value of $<30$ was used, which indicates severe fatigue. Analysis of BAFF SNPs in association with fatigue levels was performed by the online platform SNPStats.

Results: The frequency of T/T genotype of both rs9514828 and rs1224141 was reduced in primary SS patients with severe fatigue compared to those without 